\title{
THE BENNEQUIN NUMBER OF $n$-TRIVIAL CLOSED $n$-BRAIDS IS NEGATIVE
}

\author{
Oliver T. Dasbach and XiaO-Song Lin
}

\begin{abstract}
A famous result of Bennequin states that for any braid representative of the unknot the Bennequin number is negative. We will extend this result to all $n$-trivial closed $n$-braids. This is a class of infinitely many knots closed under taking mirror images. Our proof relies on a non-standard parameterization of the HOMFLY polynomial. Another interesting corollary of this parameterization is that if all Vassiliev invariants up to degree $c$ vanish on a knot of crossing number $c$, then this knot has trivial HOMFLY polynomial.
\end{abstract}

\section{Introduction}

The theory of Vassiliev knot invariants leads to a decreasing filtration on the set of knots. On the other hand, every complexity measurement on knots, such as the crossing number or the braid index or the minimal genus, gives rise to an increasing filtration. Although the interplay between these filtrations in opposite directions on the set of knots is still fairly unclear, the results of this paper show that such an interplay shall have a rich content.

In his seminal paper [Ben83], Bennequin proved that for any representative of the unknot as a closed $n$-braid with exponent sum $e$, it always holds that $|e|<n$. Bennequin's framework for the proof of this inequality was the study of contact structures on the 3-space. A smooth knot, transverse to the standard contact structure on the 3 -space, has a well-defined self-linking number. This self-linking number is an invariant of transversal knot types. Bennequin showed that every transversal knot is transversal isotopic to a closed braid. In the case when a closed braid $\hat{\mathfrak{b}}$ is considered as a transverse knot, this self-linking number is equal to the Bennequin number

$$
\beta_{t}(\mathfrak{b}):=e-n \text {. }
$$

Bennequin's inequality $|e|<n$ for the unknot also follows from work of Morton [Mor86] and independent work of Franks and Williams [FW87]. They showed that for a closed braid $\hat{\mathfrak{b}}$ the Bennequin number is always less than the lowest degree of $v$ in the HOMFLY polynomial. Since the HOMFLY polynomial of an unknot is identically 1 , the Bennequin number is negative in this case.

Received November 16, 2000.

The second author was partially supported by the Overseas Youth Cooperation Research Fund of NSFC. 
Our aim is to show that Bennequin's inequality has a natural finite type generalization. We show that an $n$-trivial closed $n$-braid has negative Bennequin number (Theorem 3.1). Since taking the mirror image preserves the $n$-triviality, we actually have $|e|<n$ for an $n$-trivial closed $n$-braid. From the point of view of the theory of Vassiliev knot invariants, the reason behind this inequality is that algebraically independent Vassiliev invariants become dependent on each other when the braid index is fixed.

One motivation of investigating such an interplay between Vassiliev invariants and the increasing filtration on the set of knots given by the braid index is best described in terms of complexity theory of Vassiliev invariants coming from the HOMFLY polynomial.

As shown by Bar-Natan [BN95b], for a knot with a diagram of $c$ crossings the computational complexity for evaluating a given Vassiliev invariant of order $k$ at this knot is in $O\left(c^{k}\right)$. Moreover, as it was proved in [JVW90] the evaluation of the Jones polynomial at all but eight points is \#P-hard. Recall that \#P is a class of counting problems that are at least as difficult as the NP-complete problems.

Since the Jones polynomial is a certain evaluation of the HOMFLY polynomial, this result carries over to the HOMFLY polynomial. There are points where the evaluation of the HOMFLY polynomial is \#P-hard.

Assuming that $P \neq N P$, this implies that for those Vassiliev invariants, which come from the HOMFLY polynomial, there cannot be an upper bound $k$ such that the computation of each of them is possible in $O\left(c^{k}\right)$-time.

However, the HOMFLY polynomial has a definition in terms of a trace of a certain representation of the braid groups [Jon87]. This means that for a fixed braid index, the HOMFLY polynomial is computable in polynomial time in the word length of the braids.

Therefore, the braid index must lead to some restrictions for Vassiliev invariants involving the word length (exponent sum). Some of them are outlined below. Some others are described in [DLL01].

Another interesting corollary of our parameterization of the HOMFLY polynomial is that if all Vassiliev invariants up to degree $c$ vanish on a knot of crossing number $c$, then this knot has trivial HOMFLY polynomial.

The first author would like to thank Joan Birman for many discussions on the topics of this paper.

\section{Parameterizing the HOMFLY polynomial as an honest two variable polynomial}

We start with a certain, non-standard definition of the HOMFLY polynomial, which seems to be more useful from the Vassiliev theory point of view. 
THE BENNEQUIN NUMBER OF $n$-TRIVIAL CLOSED $n$-BRAIDS IS NEGATIVE 631

Definition 2.1. For a braid $\mathfrak{b} \in B_{n}$ let a polynomial $\Gamma=\Gamma(\mathfrak{b}) \in \mathbb{Z}[\mu, z]$ be defined by

$$
\begin{aligned}
\Gamma\left(\mathfrak{b} \sigma_{i}\right)-\Gamma\left(\mathfrak{b} \sigma_{i}^{-1}\right) & =z \Gamma(\mathfrak{b}) \\
\Gamma\left(\mathfrak{b} \sigma_{n}^{-1}\right) & =(1-\mu z) \Gamma(\mathfrak{b}) \\
\Gamma\left(\mathfrak{b} \sigma_{n}\right) & =\Gamma(\mathfrak{b}) \\
\Gamma\left(i d_{n}\right) & =\mu^{n-1}
\end{aligned}
$$

and $\Gamma(\mu, z)$ is invariant under conjugation and relations in the braid group.

Remark 2.2. The polynomial $\Gamma$ is well-defined by the following relation with the HOMFLY polynomial. For a knot $\hat{\mathfrak{b}}$ that is the closure of a braid $\mathfrak{b} \in B_{n}$ the relation with the HOMFLY polynomial $P(v, z)$ (see e.g. [Lic88]) is given by:

$$
\left.\left((1-\mu z)^{(e-n+1) / 2} \Gamma(\mathfrak{b})(\mu, z)\right)\right|_{\mu=\frac{1-v^{2}}{z}}=P(\hat{\mathfrak{b}})(v, z) .
$$

Here, $e$ denotes the exponent sum of $\mathfrak{b}$ with respect to the standard generators of the braid group.

Proofs of properties of the polynomial $\Gamma(\mu, z)$ rely on the following way to compute it: If we have a braid $\mathfrak{b}$ which closes to a knot then we unknot the knot by crossing changes. The term on the right hand-side of the skein relation (1) corresponds to a link of two components. For a braid $\mathfrak{b}$ which closes to a link with $c$ components, we unlink it, i.e. we change crossings in two different components. The term on the right-hand side of Equation (1) is then a link with $c-1$ components.

Proposition 2.3. The polynomial $\Gamma(\mu, z)=\sum_{j} p_{j}(\mu) z^{j}$ has the following properties, which are easily checked:

1. For a split link $L=L_{1} \cup L_{2}$, i.e. $L_{1}$ and $L_{2}$ are unlinked,

$$
\Gamma(L)=\mu \Gamma\left(L_{1}\right) \Gamma\left(L_{2}\right) \text {. }
$$

2. The degree in $\mu$ of $\Gamma(\mathfrak{b})$ for $\mathfrak{b} \in B_{n}$ is less than $n$, i.e. each of the $p_{j}(\mu)$ 's is a polynomial of degree less than $n$.

3. The degree in $z$ of $\Gamma(\mathfrak{b})$ is less or equal to the word length of $\mathfrak{b}$.

4. The evaluation $\Gamma(\mathfrak{b})(0, z)$ is the Alexander polynomial, in its Conway form, of the closed braid $\hat{\mathfrak{b}}$.

5. The coefficient of $z^{k}$ in $\Gamma(\mathfrak{b})(\mu, z)$, i.e. $p_{k}$ is a Vassiliev knot invariant of order $k$ for the (framed) closed braid $\hat{\mathfrak{b}}$. This follows in the same way as outlined in [BL93, BN95a] for related polynomials.

Furthermore we have:

Proposition 2.4. Assume the braid $\mathfrak{b} \in B_{n}$ closes to a link of c components.

1. The coefficient $p_{j}(\mu)$ is an odd polynomial if $j+c$ is even, otherwise $p_{j}(\mu)$ is an even polynomial.

2. $\Gamma(\mu, 0)=p_{0}(\mu)=\mu^{c-1}$. In particular $p_{0}(\mu)=1$ if the braid closes to a knot. 
3. If the braid closes to a knot then

$$
p_{1}(\mu)=\frac{e-n+1}{2} \mu,
$$

where $e$ is the exponent sum of the braid $\mathfrak{b}$ with respect to the standard generators of the braid group.

4. For a braid which closes to a knot, the polynomial $p_{j}(\mu)$ is of degree less than or equal to $j$.

Proof. All claims follow by an easy induction. Using the skein relation (1) we express a link as the sum or difference of two links which are either of shorter word length or are "less linked".

\section{Closed $n$-braids which are $n$-trivial}

We call a knot $n$-trivial if all Vassiliev invariants up to degree $n$ vanish on it. The unknot is $n$-trivial for each $n$. It is not known whether there is another knot with this property. The Volume conjecture (see e.g. [MM01]) indeed would imply that there is none. Even for the Jones polynomial there is no example of a non-trivial knot with trivial Jones polynomial known (see e.g. [DH97]).

Theorem 3.1. Let $K$ be a n-trivial knot which is given as a closed $n$-braid $\hat{\mathfrak{b}}$. Then the Bennequin number $\beta_{t}(\mathfrak{b})$ is negative.

Proof. Let $\mathfrak{b}, \mathfrak{b} \in B_{n}$ be a braid with exponent sum $e$ satisfying our conditions. The polynomial $\Gamma(\mathfrak{b})(\mu, z)$ can be written as

$$
\Gamma(\mu, z)=\sum p_{i}(\mu) z^{i}
$$

for some polynomials $p_{i}(\mu)$ of degree less than $n$ in $\mu$.

Now the coefficients of $z^{k}$ in

$$
(1-\mu z)^{(e-n+1) / 2} \Gamma(\mu, z)
$$

is a (framing independent) Vassiliev invariant, which depends on $\mu$ and $e$.

Since, by our condition, all Vassiliev invariants up to degree $n$ vanish, we know that $p_{i}(\mu)$ must be the coefficient $c_{i}(\mu, e)$ of $z^{i}$ in $(1-\mu z)^{-(e-n+1) / 2}$.

We know that $p_{i}(\mu)$ must have degree less than $n$, but $c_{n}(\mu, e)$ has degree $n$. Thus $c_{n}(\mu, e)$ must be trivial for all choices of $\mu$, which means, $e$ has to be a root of $c_{n}(\mu, e)$.

Hence, by applying the Taylor expansion formula to $(1-\mu z)^{-(e-n+1) / 2}$, we conclude that $e$ is one of the numbers $n-1, n-3, \ldots, n-2 n+1=-n+1$.

Remark 3.2. (Unfortunately) the fact that a closed $n$-braid is $n$-trivial does not imply that it is trivial or even that its HOMFLY polynomial is trivial. By the work of Stanford [Sta96] if two braids differ by an element in the $(k+1)$-th term of the lower central series of the pure braid group $P_{n}$ then all Vassiliev invariants up to degree $k$ coincide. Now, take e.g. the braid group $B_{3}$ on 3 strands. The pure braid group $P_{3}$ is the direct product of the center of $B_{3}$ and a free group of rank 2, generated by $\sigma_{1}^{2}$ and $\sigma_{2}^{2}$. (This is a special case of Newworld's Lemma 
[DM99].) By the classification of links given as closed 3-braids [BM93] the closure of the concatenation of the braid $\sigma_{1} \sigma_{2}$ with an element in the $(k+1)$-th term of the lower central series is a non-trivial knot. By Stanford's work, it is $k$-trivial, though. Since the Jones polynomial of a closed 3-braids is identically 1, if and only if the knot is trivial [Bir85], it follows that the Jones polynomial of this link and thus the HOMFLY polynomial is non-trivial.

\section{Dimensions of HOMFLY subspaces restricted to braids}

We would like to point out one fact which becomes more apparent in the framed and reparameterized version $\Gamma(\mu, z)$ of the HOMFLY polynomial. Vassiliev invariants are dual to the space of knots. Therefore, one can ask about the dimension of Vassiliev invariants on subsets of knots.

We get

Proposition 4.1. 1. The space of (framed) Vassiliev knot invariants coming from the coefficient of the (framed) HOMFLY polynomial $\Gamma(\mu, z)$ has dimension $\left\lfloor\frac{k}{2}\right\rfloor+1$ in degree $k$.

2. Restricted to closed braids in $B_{n}$ one gets for the dimension of Vassiliev invariants in degree $k$ :

(a) If $k<n$ then the dimension is $\left\lfloor\frac{k}{2}\right\rfloor+1$.

(b) If $k \geq n$ and $n$ is odd and $k$ is even then the dimension is $\left\lfloor\frac{n}{2}\right\rfloor+1$. In all other cases it is $\left\lfloor\frac{n}{2}\right\rfloor$.

Proof. That the given dimensions form an upper bound, follows from Proposition 2.3 (2) and Proposition 2.4 (1) and (4).

It remains to construct the following: Let $k$ be the degree. We will show that there are braids in $B_{k+1}$ such that the dimension of the coefficients of $z^{k}$ is $\left\lfloor\frac{k}{2}\right\rfloor+1$.

For a braid $\mathfrak{b} \in B_{j}$ the polynomial $\Gamma(\mu, z)$ satisfies:

$$
\Gamma\left(\mathfrak{b} \sigma_{j}^{3}\right)(\mu, z)=\left(1+z \mu+z^{2}\right) \Gamma(\mathfrak{b})(\mu, z) .
$$

Furthermore, we have for arbitrary $k$ :

$$
\Gamma\left(\sigma_{1}^{-1} \sigma_{2}^{-1} \ldots \sigma_{k}^{-1}\right)(\mu, z)=(1-\mu z)^{k} .
$$

In particular, the coefficient of $z^{k}$ is $\pm \mu^{k}$.

Combining these two formulas, one sees that the coefficient of $z^{k}$ in

$$
\Gamma\left(\sigma_{1}^{-1} \sigma_{2}^{-1} \ldots \sigma_{k-2}^{-1} \sigma_{k-1} \sigma_{k}^{3}\right)(\mu, z)
$$

is $\pm \mu^{k-2}$. Replacing successively pairs $\sigma_{j-1}^{-1} \sigma_{j}^{-1}$ by $\sigma_{j-1} \sigma_{j}^{3}$, where $j$ has the same parity as $k$, yields polynomials with coefficients of $z^{k}$ equal to $\pm \mu^{j-2}$. $k$.

Hence the dimension is equal to the number of $j \leq k$ with the same parity as

Remark 4.2. Part (1) of the previous proposition should also follow along the lines of [Men97]. 


\section{Knots whose evaluation at Vassiliev invariants coincide}

Our reparametrization allows us to give an improvement of a theorem in [KSS97]. The general question is: Given a quantum polynomial and a knot $K$ of crossing number $c$, up to which degree - as a function in $c$ - does one has to know the values of Vassiliev invariants at the knot $K$, so that the whole polynomial is already determined? Since there are only finitely many knots of crossing number $c$, such a function has to exist. It is not clear, however, whether this function has some nice form. In [KSS97] it was shown that for the HOMFLY polynomial such a function is bounded by a quadratic polynomial in $c$. We will show, that it is in fact bounded by $c$. Why is this important? It is still unknown whether Vassiliev invariants can distinguish knots. The number of knots of crossing number $c$ grows exponentially [Wel92]. On the other hand, the best lower bound known for the dimensions of Vassiliev invariants of degree $c$ is exponential in the square root of $c$ [Das00]. This might indicate that the space of Vassiliev invariants is simply not big enough to distinguish knots.

Theorem 5.1. Let the knots $\hat{\mathfrak{b}}_{1}$ and $\hat{\mathfrak{b}}_{2}$ be closures of braids of length less or equal than $c$, for some number $c$.

If the evaluation of all Vassiliev invariants up to order c coincide on $\hat{\mathfrak{b}}_{1}$ and $\hat{\mathfrak{b}}_{2}$ then their HOMFLY polynomials are equal.

Proof. By Proposition 2.3 (3) we know that the polynomials $\Gamma\left(\mathfrak{b}_{1}\right)$ and $\Gamma\left(\mathfrak{b}_{2}\right)$ are polynomials in $z$ of degree less than or equal to $c$.

Let $e_{1}\left(e_{2}\right.$, respectively) be the exponent sum of the braid $\mathfrak{b}_{1}\left(\mathfrak{b}_{2}\right.$, respectively) on $n_{1}$ ( $n_{2}$, respectively) strands.

We know that the HOMFLY polynomial $P(\mu, z)$ - with our reparameterization - is given by a power series in $z$

$$
P\left(\hat{\mathfrak{b}}_{1}\right)(\mu, z)=(1-\mu z)^{(e-n+1) / 2} \Gamma\left(\mathfrak{b}_{1}\right)(\mu, z) .
$$

The coefficient of $z^{j}$ in $P\left(\hat{\mathfrak{b}}_{1}\right)(\mu, z)$ is a Vassiliev invariant of order $j$.

Since $\Gamma\left(\hat{\mathfrak{b}}_{1}\right)(\mu, z)$ is of degree in $z$ less than or equal to $c, \Gamma\left(\mathfrak{b}_{1}\right)(\mu, z)$ is determined by all Vassiliev invariants up to order $c$. In turn, $P\left(\hat{\mathfrak{b}}_{1}\right)(\mu, z)$ itself is determined by all terms in $z^{j}$ of degree less than or equal to $c$.

The same argument works with $\mathfrak{b}_{2}$ and we are done.

Remark 5.2. For the definition of $\Gamma$ we do not really need the knot given as a closed braid. We can work with arbitrary knot diagrams of framed knots instead. Thus, in the last theorem we can replace the word length by the crossing number. As a corollary we get:

Corollary 5.3. If all Vassiliev invariants up to degree $c$ vanish on a knot $K$ of crossing number $c$ then $K$ has trivial HOMFLY polynomial.

\section{References}

[Ben83] D. Bennequin, Entrelacement et équations de Pfaff, (French) Astérisque, 107-108, Soc. Math. France, Paris, 1983. 
[Bir85] J. S. Birman, On the Jones Polynomial of Closed 3-braids, Invent. Math. 81 (1985), $287-294$.

[BL93] J. S. Birman and X.-S. Lin, Knot polynomials and Vassiliev's invariants, Invent. Math. 111 (1993), no. 2, 225-270.

[BM93] J. S. Birman and W.W. Menasco, Studying links via closed braids III. Classifying links which are closed 3-braids, Pacific J. Math. 161 (1993), no. 1, 25-113.

[BN95a] D. Bar-Natan, On the Vassiliev knot invariants, Topology 34 (1995), no. 2, 423-472.

[BN95b] D. Bar-Natan, Polynomial invariants are polynomial, Math. Res. Lett. 2 (1995), no. 3, 239-246.

[Das00] O.T. Dasbach, On the combinatorial structure of primitive Vassiliev invariants III - A lower bound, Commun. Contemp. Math. 2 (2000), no. 4, 579-590.

[DH97] O.T. Dasbach and S. Hougardy, Does the Jones Polynomial Detect Unknottedness?, Experiment. Math. 6 (1997), no. 1, 51-56.

[DLL01] O.T. Dasbach, T.D. Le, and X.-S. Lin, Quantum morphing and the Jones polynomial, to appear in Commun. Math. Physics, 2001.

[DM99] O.T. Dasbach and B. Mangum, The automorphism group of a free group is not subgroup separable, to appear in: Knots, Braids, and Mapping Class Groups Conference Proceedings.

[FW87] J. Franks and R. F. Williams, Braids and the Jones polynomial, Trans. Amer. Math. Soc. 303 (1987), no. 1, 97-108.

[Jon87] V. F. R. Jones, Hecke algebra representations of braid groups and link polynomials, Ann. of Math. (2) 126 (1987), no. 2, 335-388.

[JVW90] F. Jaeger, D. L. Vertigan, and D. J. A. Welsh, On the computational complexity of the Jones and Tutte polynomials, Math. Proc. Cambridge Philos. Soc. 108 (1990), no. 1, 35-53.

[KSS97] L. H. Kauffman, M. Saito, and S.F. Sawin, On finiteness of certain Vassiliev invariants, J. Knot Theory Ramifications 6 (1997), no. 2, 291-297.

[Lic88] W.B.R. Lickorish, Polynomials for links, Bull. London. Math. Soc. 20 (1988), 558588.

[Men97] G. Meng, Bracket models for weight systems and the universal Vassiliev invariants, Topology Appl. 76 (1997), no. 1, 47-60.

[MM01] H. Murakami and J. Murakami, The colored Jones polynomials and the simplicial volume of a knot, Acta Math. 186 (2001), no. 1, 85-104.

[Mor86] H. R. Morton, Seifert circles and knot polynomials, Math. Proc. Cambridge Philos. Soc. 99 (1986), 107-109.

[Sta96] T. Stanford, Braid commutators and Vassiliev invariants, Pacific J. Math. 174 (1996), no. 1, 269-276.

[Wel92] D. J. A. Welsh, On the number of knots and links, Sets, graphs and numbers (Budapest, 1991), 713-718, Colloq. Math. Soc. Jnos Bolyai, 60, North-Holland, Amsterdam, 1992.

Oklahoma State University, Department of Mathematics, Stillwater, OK 74078 1058.

E-mail address: kasten@math.okstate.edu

University of California, Riverside, Department of Mathematics, Riverside, CA $92521-0135$.

E-mail address: xl@math.ucr.edu 